\title{
Damage Correlation between in situ Ion Irradiation and Neutron Irradiation in Molybdenum
}

\author{
Marquis Kirk*, Meimei Li**, and Peter Baldo* \\ *Materials Science Division, Argonne National Laboratory, Argonne, IL 60439. \\ **Nuclear Engineering Division, Argonne National Laboratory, Argonne, IL 60439.
}

It has long been hoped that computer modeling can accurately predict responses in materials over many years of neutron irradiation at elevated temperatures. Although much progress has been achieved in the development of the modeling techniques, experiments involving neutron irradiation to confirm model predictions are relatively few and usually quite difficult with a long turnaround time. To solve this experimental difficulty, we are pursuing a new direction to utilize the IVEMTandem Facility at ANL to benchmark computer models for irradiation of materials.

In this new effort, we recently employed $1 \mathrm{MeV} \mathrm{Kr}$ ion irradiation in situ in our TEM facility to produce and image defect clusters (generally dislocation loops) at controlled temperatures, over a range of doses and dose rates, and over a range of sample thicknesses in well characterized molybdenum thin foils. The essential objectives of this experiment are to measure and characterize the formation of defect microstructure as functions of these parameters (all of which can be modeled by computer simulation), compare to neutron irradiation data on the same material [1], and demonstrate the methods of extrapolation from ion irradiations at high dose rate in thin samples, to neutron irradiation at low dose rate in bulk samples. Ideally, if successful, an ion irradiation and electron microscopy of a material in our facility over a day or two can result, through modeling, in predictions of microstructural behavior and property changes over several years, even decades, of neutron irradiation in fission or fusion reactors.

Data consisting of defect cluster densities were obtained in pure Mo foils irradiated in situ to a series of doses and imaged using weak-beam dark-field with a constant diffraction condition, such that defect areal density could be measured as a function of thickness over the wedged foil. Separate experiments were performed at three temperatures, 20 and $80^{\circ} \mathrm{C}$, which are just below stage three where only interstitials have high mobility, and $300^{\circ} \mathrm{C}$, which is just above stage three where both interstitials and vacancies are highly mobile. In addition, data was obtained at three dose rates at $80^{\circ} \mathrm{C}$.

An example of the image results is shown in Figure 1. An example of quantitative results in Figure 2 demonstrates the different dependencies of defect cluster density on dose and foil thickness at 80 and $300^{\circ} \mathrm{C}$.

A next step in this experimental program is to obtain tomographic data to measure defect density as a function of foil depth and thus quantify the effects of nearby surfaces on point defect clustering. Finally, we hope to make a detailed comparison with computer simulations [2] constructed to match our various experimental conditions and extensive data on defect cluster density as functions of sample thickness, ion dose and dose rate, and irradiation temperature. 
References

[1] Meimei Li, M. Eldrup, T. S. Byun, N. Hashimoto, L. L. Snead, S. J. Zinkle, J. Nucl. Mater. $376(2008) 11$.

[2] M. J. Caturla, N. Soneda, E. Alonso, B.D. Wirth, T. Dõaz de la Rubia, J.M. Perlado, J. Nucl. Mater. 276 (2000) 13.

In-situ $1 \mathrm{MeV} \mathrm{Kr}$ ion irradiation at $80^{\circ} \mathrm{C}\left(5 \times 10^{-4} \mathrm{dpa} / \mathrm{sec}\right)$
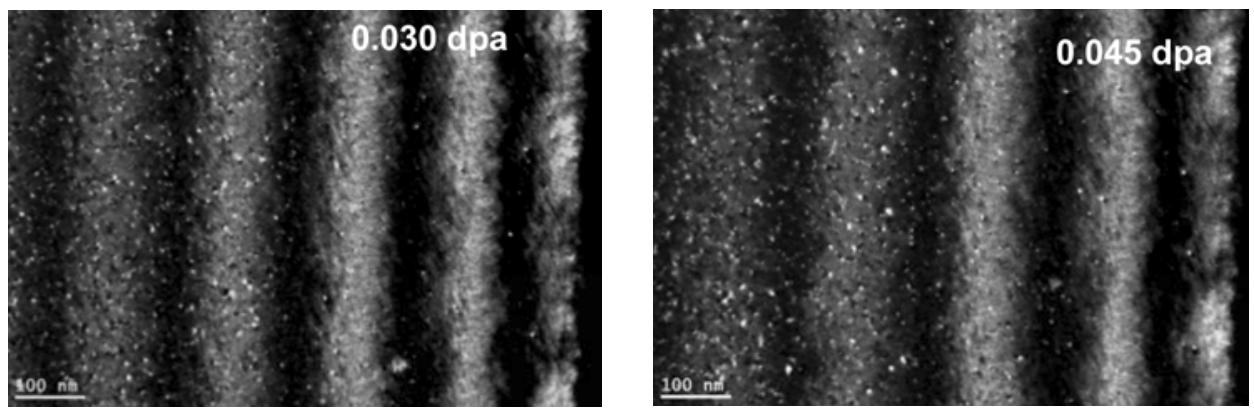

\section{Neutron irradiation at $80^{\circ} \mathrm{C}$ in $\operatorname{HFIR}\left(3 \times 10^{-7} \mathrm{dpa} / \mathrm{sec}\right)$}
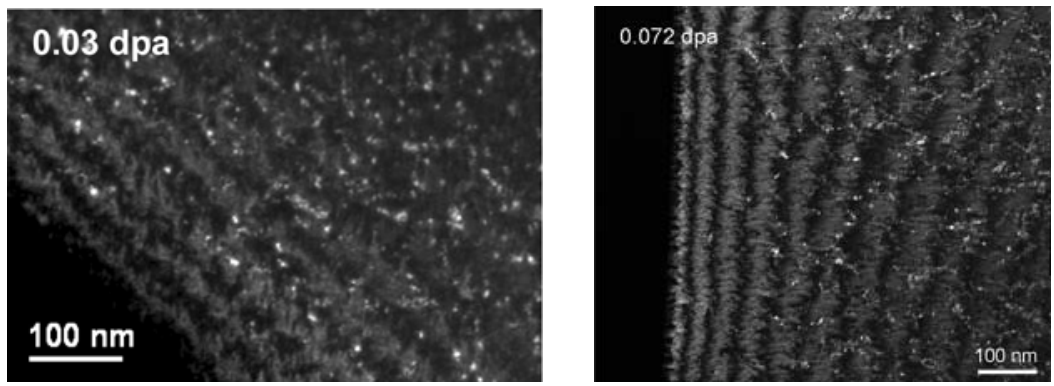

FIG. 1. Comparison of defect clusters between ion and neutron irradiations in molybdenum.
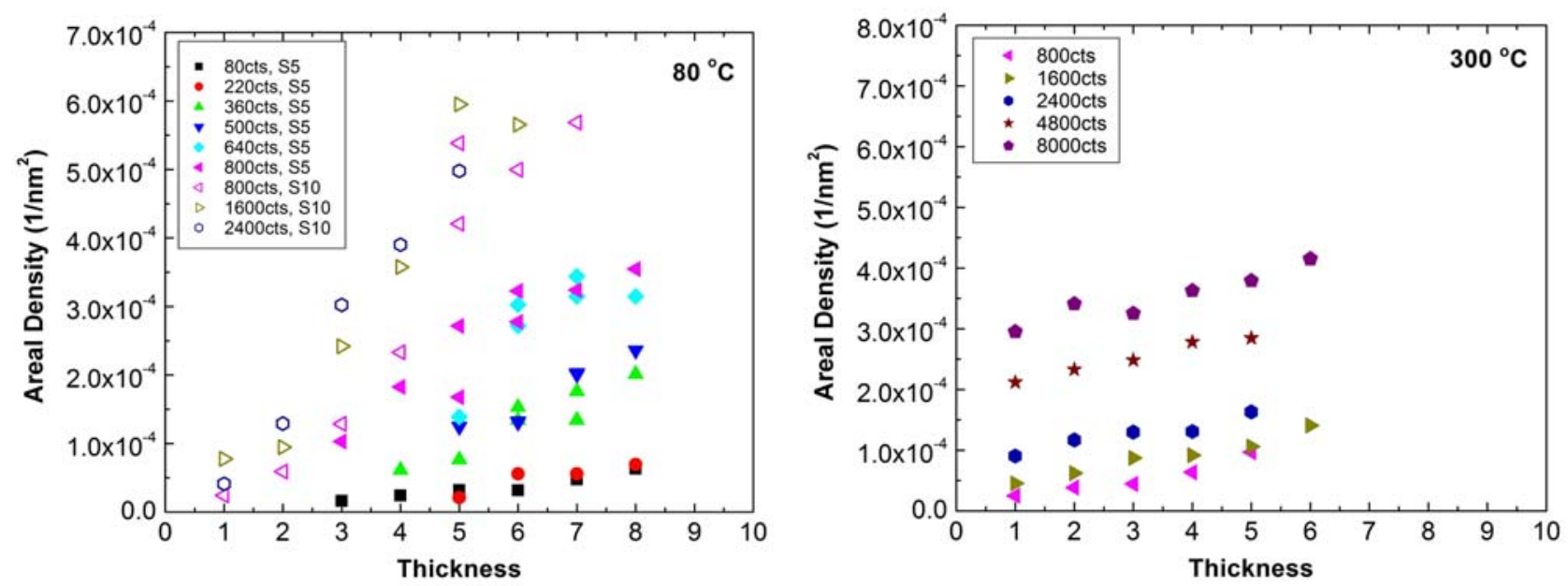

FIG. 2. Comparison of defect cluster density as functions of dose and foil thickness at 80 and $300^{\circ} \mathrm{C}$ in molybdenum. Thickness is given in the number of extinction contours; dose in counts scales as 800 counts $=0.015$ dpa. 